\title{
Experimental tests of multiplicative Bell inequalities and the fundamental role of local correlations
}

\author{
Dilip Paneru $\odot,{ }^{1}$ Amit Te'eni, ${ }^{2}$ Bar Y. Peled,${ }^{3}$ James Hubble, ${ }^{1}$ Yingwen Zhang, ${ }^{4}$ \\ Avishy Carmi $\odot{ }^{3}$ Eliahu Cohen $\odot,{ }^{2, *}$ and Ebrahim Karimi ${ }^{1,4}$ \\ ${ }^{1}$ Department of Physics, University of Ottawa, 25 Templeton Street, Ottawa, Ontario K1N 6N5, Canada \\ ${ }^{2}$ Faculty of Engineering and the Institute of Nanotechnology and Advanced Materials, Bar Ilan University, Ramat Gan 5290002, Israel \\ ${ }^{3}$ Center for Quantum Information Science and Technology \& Faculty of Engineering Sciences, \\ Ben-Gurion University of the Negev, Beersheba 8410501, Israel \\ ${ }^{4}$ National Research Council of Canada, 100 Sussex Drive, Ottawa, Ontario K1A0R6, Canada
}

(Received 15 September 2020; accepted 9 February 2021; published 12 March 2021)

\begin{abstract}
Bell inequalities are mathematical constructs that demarcate the boundary between quantum and classical physics. A new class of multiplicative Bell inequalities originating from a volume maximization game (based on products of correlators within bipartite systems) has been recently proposed. For these new Bell parameters, it is relatively easy to find the classical and quantum, i.e., Tsirelson, limits. Here, we experimentally test the Tsirelson bounds of these inequalities using polarization-entangled photons for a different number of measurements $(n)$, each party can perform. For $n=2,3,4$, we report the experimental violation of local hidden variable theories. In addition, we experimentally compare the results with the parameters obtained from a fully deterministic strategy, and observe the conjectured nature of the ratio. Finally, utilizing the principle of "relativistic independence" encapsulating the locality of uncertainty relations, we theoretically derive and experimentally test new and richer quantum bounds for both the multiplicative and the additive Bell parameters for $n=2$. Our findings strengthen the deep correspondence between local and nonlocal correlations, and pave the way for both theoretical (e.g., better understanding of nonlocal correlations) and practical (e.g., Bell tests and quantum technologies with inefficient detectors) applications.
\end{abstract}

DOI: 10.1103/PhysRevResearch.3.L012025

\section{INTRODUCTION}

Ever since quantum mechanics was introduced to describe the subatomic world, the foundational aspects, most notably the nondeterministic nature of experimental outcomes, have always been a topic of discussion among physicists and philosophers [1]. In their seminal work [2], Einstein, Podolsky, and Rosen (EPR) argued for the incompatibility of quantum theory with the idea of local realism. Since then, attempts were made to incorporate extra parameters within the theory, the so-called hidden variables, to "complete" the quantum formalism [3]. However, in 1964 John Bell showed that there exist experiments for which any local hidden variable theory must disagree with quantum mechanics about the predicted outcome [4]. This discrepancy is most conveniently illustrated by Bell parameters, i.e., measurable quantities whose values must be bounded to a certain extent in any local hidden variable theory, but can exceed these bounds according to quantum mechanics [5]. Experiments carried out to test these inequalities [6-9] have always vindicated quantum

\footnotetext{
*eliahu.cohen@biu.ac.il

Published by the American Physical Society under the terms of the Creative Commons Attribution 4.0 International license. Further distribution of this work must maintain attribution to the author(s) and the published article's title, journal citation, and DOI.
}

mechanics, thereby showing that local realistic theories do not present an adequate representation of the physical world. Recent new experiments have also significantly progressed towards closing loopholes in a typical Bell experiment, such as freedom-of-choice, fair-sampling, communication (or locality), coincidence, and memory loopholes [10-16]. Several works have attempted to find the extent of Bell parameters involving quantum correlations [17-19]. However, finding the classical and quantum (Tsirelson) bounds of these expressions in general remains a challenging task $[18,19]$. Recently, Bell parameters with products of correlators between random variables shared between two parties, namely Alice and Bob, were introduced [20] which alleviate the above difficulties. Corresponding to the number of random variables $n$, measurable by each of the parties, the multiplicative Bell parameter would be proportional to a certain volume in the $n$-dimensional space (this was shown to result from a specific coordination game Alice plays with Bob). For the simplest case where Alice and Bob measure two random variables each, it was also proven that the bound for classical correlations is strictly less than that for quantum correlations. Moreover, these multiplicative Bell inequalities were shown to be more robust to detector inefficiency than the additive ones [20], which is another useful property of the proposed nonlinear inequalities. In general, the Tsirelson bound for the multiplicative Bell parameters $\mathcal{B}_{n}$, corresponding to measurement $n$, was shown to be $\left|\mathcal{B}_{n}\right| \leqslant n$ !. The Tsirelson bounds were derived from the structure of the quantum covariance matrix [21], under the assumption of 
"relativistic independence" [22], mathematically expressing the requirement for locality of uncertainty relations. With this assumption, which we now put to experimental test, it was possible to derive the strength of quantum correlations from first principles and also influence some other research directions [23-26]. Furthermore, the nonlinear nature of the proposed inequalities may shed light on the nonpolytopic structure of the set of quantum correlations. Here, we present the results of experiments testing the Tsirelson bounds for the proposed multiplicative inequalities for various $n$ values, and observe their surprising large $n$ behavior. Moreover, based on relativistic independence we propose and put to test new Tsirelson bounds that are richer than those derived in the past. This shows the interplay between local and nonlocal correlations, which has both fundamental and applicative implications.

\section{THEORY}

Let us consider a photon pair entangled in the polarization degree of freedom,

$$
|\psi\rangle=\frac{1}{\sqrt{2}}\left(|H\rangle_{A}|V\rangle_{B}-|V\rangle_{V}|H\rangle_{B}\right),
$$

where $|H\rangle$ and $|V\rangle$ stand for horizontal and vertical linear polarization states, and the subscripts $A$ and $B$ represent photon states for Alice and Bob, two spatially separated observers. In the multiplicative Bell scenario, the observers have a different Bell parameter depending upon the number $n$ of different measurements that each can perform. For a general $n$ the Bell parameter is defined as

$$
\begin{aligned}
\mathcal{B}_{n} & =\prod_{j=1}^{n} v_{j} \cdot c_{j} \\
& =\left(c_{1 n}+\cdots+c_{n n}\right) \prod_{j=1}^{n-1}\left(c_{1 j}+\ldots+c_{j j}-j c_{j+1, j}\right),
\end{aligned}
$$

where $c_{i j}=c_{a_{i} b_{j}}$ is the expectation value of the polarization measurements along $a_{i}$ and $b_{j} . c_{j}$ is the vector comprising all $c_{i j}$ for a fixed value of $j$, and $v_{j}$ is the $j$ th column of the matrix:

$$
V=\left[\begin{array}{ccccc}
1 & 1 & \ldots & 1 & 1 \\
-1 & 1 & \cdots & 1 & 1 \\
& -2 & \ddots & \vdots & 1 \\
& & \ddots & 1 & \vdots \\
& & & -(n-1) & 1
\end{array}\right] .
$$

A strategy to select the vectors $a_{1} \ldots a_{n}$ and $b_{1} \ldots b_{n}$, so as to saturate the Tsirelson bounds $\left(\left|\mathcal{B}_{n}\right| \leqslant n !\right)$ is outlined in [20]. The multiplicative Bell parameters, $\mathcal{B}_{n} \triangleq \prod_{j=1}^{n} v_{j} \cdot c_{j}$, can be associated with the additive Bell parameters, $B_{n}^{\prime} \triangleq \sum_{j=1}^{n} v_{j}$. $c_{j}$, using the following relation:

$$
\left|\mathcal{B}_{n}\right| \leqslant\left(\frac{\mathcal{B}_{n}^{\prime}}{n}\right)^{n},
$$

which is a result of the inequality of geometric and arithmetic means, i.e., $\sqrt[n]{\mathcal{B}_{n}}=\sqrt[n]{\prod_{j=1}^{n} v_{j} \cdot c_{j}} \leqslant \frac{\sum_{j=1}^{n} v_{j} \cdot c_{j}}{n}$. The latter expression can be used to find classical upper bounds (not necessarily tight) over $\mathcal{B}_{n}$, given the classical bounds over $\mathcal{B}_{n}^{\prime}$. Using the known fact that additive Bell inequalities are saturated by deterministic strategies [5], one may go over all such strategies and find the tight classical bounds for $\mathcal{B}_{n}^{\prime}$. The results are as follows:

$$
\begin{aligned}
& \mathcal{B}_{2}^{\prime} \leqslant 2 \Rightarrow \mathcal{B}_{2} \leqslant 1 ; \\
& \mathcal{B}_{3}^{\prime} \leqslant 5 \Rightarrow \mathcal{B}_{3} \leqslant\left(\frac{5}{3}\right)^{3} \approx 4.6 ; \\
& \mathcal{B}_{4}^{\prime} \leqslant 8 \Rightarrow \mathcal{B}_{4} \leqslant 16 .
\end{aligned}
$$

$\mathcal{B}_{2}^{\prime}$ is the well-known Bell-CHSH parameter [27]. For $n=2$ and 4, this method yields $\mathcal{B}_{2}=1$ and $\mathcal{B}_{4}=16$, which set the tight classical bounds for these particular values of $n$. This scheme does not yield a useful classical bound for $n>4$.

Since finding the classical limit for the Bell parameter, Eq. (2), is suspected to be difficult in general, an independent and deterministic strategy was proposed for both Alice and Bob [20], and the corresponding limit was calculated. In this strategy, Bob always chooses his random variable to be +1 , while Alice's choice alternates between +1 and -1 for all of her variables $A_{i}$, until $i<i_{c}$, where $i_{c}$ is some cutoff number; and for $i>i_{c}$, she chooses $A_{i}$ to be +1 . The correlations take the following values:

$$
c_{i j}=\left\{\begin{array}{ll}
(-1)^{i} & i \leqslant i_{c} \\
1 & i>i_{c}
\end{array} .\right.
$$

The value for the cutoff $i_{c}$ is taken such that it maximizes the value for the Bell parameter. Analytically, the value $\mathcal{B}_{n}$ obtained for this fully deterministic strategy, $\mathrm{FD}_{n}$, can be explicitly calculated, and its value is

$$
\mathrm{FD}_{n}=2^{i_{c}}\left[\left(\frac{i_{c}}{2}\right) !\right]^{2}\left(n-i_{c}\right) i_{c}^{\left(n-i_{c}-1\right)} .
$$

The maximal values obtained from the fully deterministic strategy for $n=2$ and 4 coincide with the classical bounds obtained in Eq. (4). The ratio of the fully deterministic strategy and Tsirelson bound, i.e., $\mathrm{FD}_{n} / n$ !, approaches $\sqrt{\pi / 2 e}$ as $n \rightarrow \infty$.

\section{EXPERIMENT}

Paired 0.5-mm-thick type-I bismuth triborate crystals (BiBO), one rotated by $90^{\circ}$ with respect to the other, are pumped by a quasicontinuous wave $100-\mathrm{mW}, 355-\mathrm{nm}$ beam to generate photon pairs (signal and idler) via spontaneous parametric downconversion (SPDC) at a degenerate wavelength of $710 \mathrm{~nm}$ [28] [see Fig. 1(a)]. A half-wave plate, placed before the paired crystals, is used to tailor the photon pair state by controlling the pump beam polarization state. Due to indistinguishability, the generated photon pairs are entangled in the polarization degree of freedom. The polarization of the pump beam and the orientation of the crystal was tuned so as to obtain the state, $|\psi\rangle_{\text {SPDC }}=\frac{1}{\sqrt{2}}\left(|H\rangle_{A}|H\rangle_{B}-|V\rangle_{A}|V\rangle_{B}\right)$. A half-wave plate was placed in one of the arms, say Bob, so that the horizontal and vertical polarization states are exchanged and the state in Eq. (1) was obtained. The $355-\mathrm{nm}$ pump beam is afterwards filtered out with a long pass filter. The photon pairs are separated by a 50:50 beamsplitter (BS), and the photons travel along two different arms. To select two diametrically opposite 


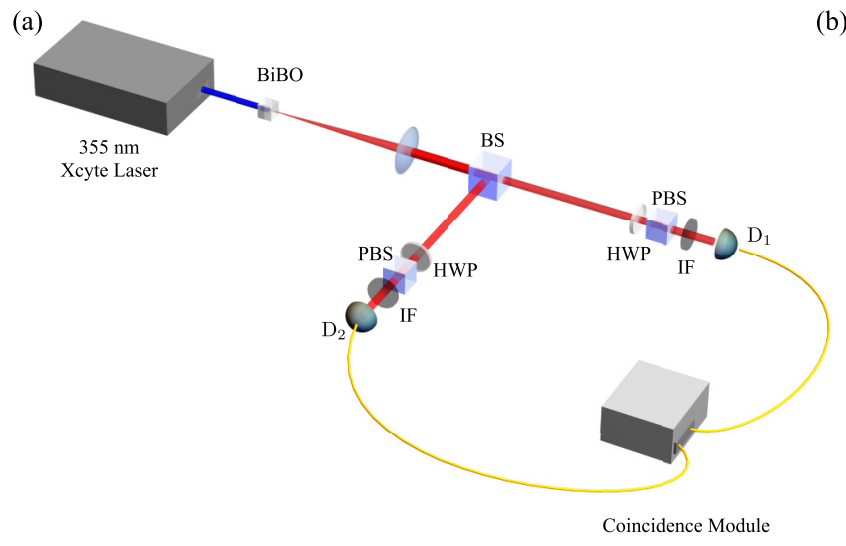

(b)

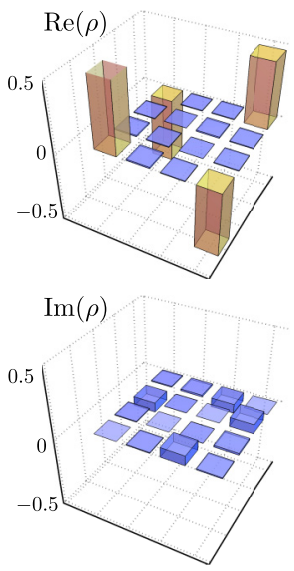

(c)

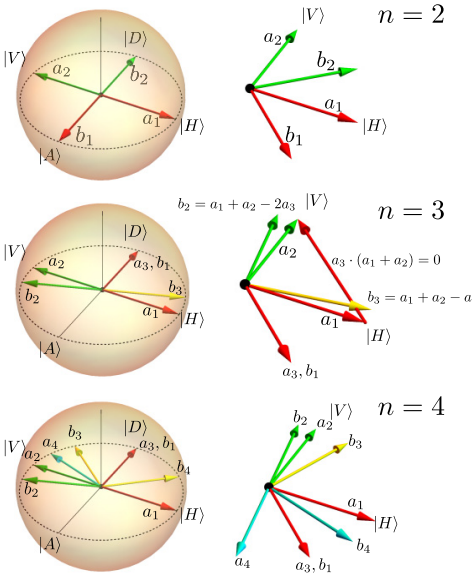

FIG. 1. Experimental scheme and the chosen strategy to perform measurement of multiplicative Bell parameters. (a) Sketch of the setup used for generating polarization entangled photons and projecting them onto the states chosen by Alice and Bob's strategies. Entangled photon pairs are generated after pumping paired BiBO (bismuth triborate) crystals, and then separated by a 50:50 beamsplitter (BS) into two arms (Alice and Bob). The polarization measurement stage on each side consists of a half-wave plate (HWP) and polarizing beamsplitter (PBS). The photons are filtered by a 710-nm interference bandpass filter (IF), and coupled into single mode fibers, and then detected using single photon avalanche diodes whose signals are sent to a coincidence module from which coincidence events can be observed. (b) Real and imaginary parts of the experimentally reconstructed density matrix of the generated entangled state are shown in the $|H\rangle_{A}|H\rangle_{B},|H\rangle_{A}|V\rangle_{B},|V\rangle_{A}|H\rangle_{B}$, and $|V\rangle_{A}|V\rangle_{B}$ basis. The generated state fidelity is $\simeq 0.977$. (c) The projective measurement strategy, i.e., $\left\{a_{1}, a_{2}, \ldots\right\}$ and $\left\{b_{1}, b_{2}, \ldots\right\}$, chosen by Alice and Bob, are shown for $n=2, n=3$, and $n=4$ on the polarization Poincaré sphere.

regions of the SPDC cone, we place a pair of irises on each arm such that they center on the required region. In each arm projective measurement of the polarization state is performed by a combination of a quarter-wave plate, a half-wave plate, and a polarizing beamsplitter (PBS). Bandpass filters of $(710 \pm 5) \mathrm{nm}$ are placed before $20 \times$ objectives so that only degenerate photon pairs are coupled into single mode fibers of core diameter $5 \mu \mathrm{m}$. Then, the photons are detected via a pair of single photon avalanche diode (SPAD) detectors (Excelitas SPCM-AQRH-14-FC), and are finally counted via a time-correlated single photon counting system. To achieve a maximally entangled state of Eq. (1), the angle of the waveplate before the crystal and the orientation of the crystal were appropriately adjusted. The coincidence rates depending upon the difference in the two half-wave plate orientations, ranged from $5220 s^{-1}$ to $34 s^{-1}$. The visibilities in the $H / V$ basis and $\pm 45^{\circ}$ linear polarization states were $(98.2 \pm 0.5) \%$ and $(97.3 \pm 0.5) \%$, respectively. Real and imaginary parts of the generated photons' density matrix are shown in Fig. 1(b). The fidelity of the generated state from the reconstructed density matrix was measured to be $\simeq 0.977$, confirming the high quality of the entangled source. Figure 1(c) illustrates the set of vectors used in projecting Alice and Bob's quantum states, i.e.. $\left\{a_{1}, a_{2}, a_{3}, \ldots\right\}$ and $\left\{b_{1}, b_{2}, b_{3}, \ldots\right\}$, on the polarization Poincaré sphere. The number of photon counts $N$ detected in coincidence along $a$ and $b$ by Alice and Bob's detectors was recorded, from which one can obtain the expectation value of the measurement via $c_{a b}=$ $\left(N_{++}-N_{+-}-N_{-+}+N_{--}\right) /\left(N_{++}+N_{+-}+N_{-+}+N_{--}\right)$, such that $N_{+-}$, for instance, refers to the joint measurement where Alice and Bob set their apparatus to measure the state along the positive and negative direction of $a$ and $b$, respectively. Using this approach, as a verification, the $\mathrm{CHSH}$ parameter for our single photon source is measured and found to be $2.748 \pm 0.026 \leqslant 2 \sqrt{2}$, which lies beyond the classical limit of 2 . The Bell parameter for $n=2$ is $\mathcal{B}_{2}=\left|\left(c_{12}+c_{22}\right)\left(c_{12}-c_{21}\right)\right|$. The classical and quantum limits of $\mathcal{B}_{2}$ are, respectively, $\mathcal{B}_{2}^{\text {Classical }} \leqslant 1$ and $\mathcal{B}_{2}^{\text {Quantum }} \leqslant 2$ !. The value for $\mathcal{B}_{2}$ as calculated from our experiment was $\mathcal{B}_{2}=1.88 \pm 0.05$, which is beyond the classical limit 1. For $n=3$ the Bell parameter is given by, $\mathcal{B}_{3}=\left|\left(c_{13}+c_{23}+c_{33}\right)\left(c_{11}-c_{21}\right)\left(c_{12}+c_{22}-2 c_{32}\right)\right|$.

The classical limit for $\mathcal{B}_{3}$ lies somewhere between 4 and 4.6. Experimentally the observed value for $\mathcal{B}_{3}$ is $5.85 \pm 0.31$. Experimentally measured values for the Bell parameters up to $n=7$ with the Tsirelson bounds and the classical limits (where applicable) are shown in Table I. The experimentally measured Bell parameters for higher values of $n$ are plotted in Fig. 2(a). The primary contribution to the uncertainties in the Bell parameter is associated with the rotation of the half-wave plates in the detection state. Since the coincidence counts were taken by rotating motor controlled waveplates at intervals of $1^{\circ}$, the corresponding maximum and the average uncertainties in the expectation values are 0.0698 and

TABLE I. Classical ( $\left.\mathcal{B}_{n}^{\text {Classical }}\right)$, fully deterministic $\mathrm{FD}_{n}$, and Tsirelson $\left(\mathcal{B}_{n}^{\text {Quantum }}\right)$ bounds for the multiplicative Bell parameters, and the experimentally $\left(\mathcal{B}_{n}^{\text {Experiment }}\right)$ measured values.

\begin{tabular}{ccccc}
\hline \hline$n$ & $\mathcal{B}_{n}^{\text {Classical }}$ & $\mathrm{FD}_{n}$ & $\mathcal{B}_{n}^{\text {Experiment }}$ & $\mathcal{B}_{n}^{\text {Quantum }}(n !)$ \\
\hline 2 & 1 & 1 & $1.88 \pm 0.05$ & 2 \\
3 & $4-4.6$ & 4 & $5.85 \pm 0.31$ & 6 \\
4 & 16 & 16 & $23.3 \pm 1.4$ & 24 \\
5 & N.A. & 64 & $115 \pm 9$ & 120 \\
6 & N.A. & 512 & $687 \pm 59$ & 720 \\
7 & N.A. & 3072 & $4655 \pm 374$ & 5040 \\
\hline \hline
\end{tabular}



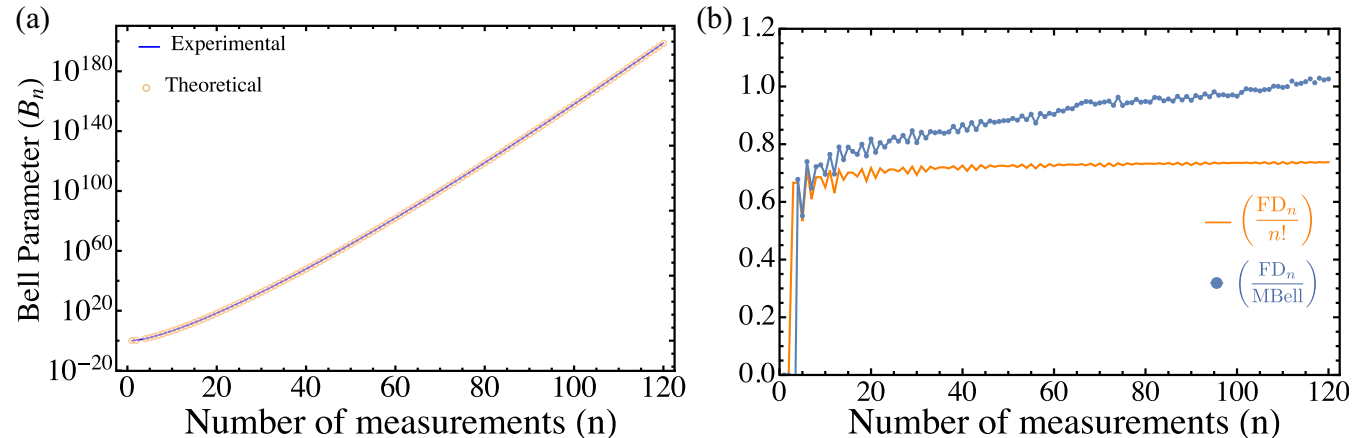

FIG. 2. Experimentally calculated multiplicative Bell parameters (MBell). (a) Logarithmic plot of the experimentally calculated Bell parameters (blue), and theoretical quantum limit of the Bell parameter, i.e., n!, (red). (b) Ratio of the parameters generated from the fully deterministic strategy $\mathrm{FD}_{n}$ taken with the Tsirelson bound $n$ ! (red), and with the experimentally observed Bell parameter MBell (blue).

0.044, respectively. The uncertainty associated with photon counting from Poissonian statistics contributes to maximum uncertainty of 0.003 . Thus the uncertainties, are dominated by the rotation of the waveplates, which is extremely small, and not visible in Fig. 2(a). The obtained results are close to the corresponding Tsirelson bounds. In order to quantify the results, we plot the ratio between the maximal quantum bound and the observed parameters [see Fig. 2(b)]. Most of the obtained results are within $80 \%$ of the maximal value. As a comparison with the fully deterministic strategy (6), the theoretical $\left(\mathrm{FD}_{n} / n\right.$ !) and the obtained experimental $\left(\mathrm{FD}_{n} / \mathrm{MBell}\right)$ ratios are shown in Fig. 2(b) for values of $n$ up to 255. As the experimentally observed Bell parameters are less than the actual ones the experimentally calculated values for the ratio are slightly larger than the theoretical ones.

\section{LOCALITY OF UNCERTAINTY AND RICHER QUANTUM BOUNDS}

Recently it was proposed [22] that the locality of uncertainty, i.e., the requirement that local uncertainties are independent of the measurement choices of any other parties, can give rise to both known and hitherto unnoticed bounds on nonlocal correlations in any statistically meaningful theory. For two parties sharing a Bell state, richer bounds for the $\mathrm{CHSH}$ parameter and the multiplicative Bell parameter will be obtained, employing also the local correlations. Let us first define the local correlation, say on Alice's side,

$$
\eta_{A}=\frac{\left\langle A_{i} A_{j}\right\rangle-\left\langle A_{i}\right\rangle\left\langle A_{j}\right\rangle}{\Delta_{A_{i}} \Delta_{A_{j}}},
$$

where $A_{i}$, and $A_{j}$ are two local observables for Alice, and $\Delta_{A_{k}}^{2}=\left\langle A_{k}^{2}\right\rangle-\left\langle A_{k}\right\rangle^{2}$. In the Appendix we show that these local correlations on Alice's side give rise to new, more elaborate bounds for the standard $\mathrm{CHSH}$ and the multiplicative Bell parameters, which, respectively, are $\mathrm{CHSH} \leqslant$ $\sqrt{2}\left(\sqrt{1+\eta_{A}}+\sqrt{1-\eta_{A}}\right)$ and $\mathcal{B}_{2} \leqslant 1+\sqrt{1-\eta_{A}^{2}}$. For the special case of maximally entangled states, as the one considered here, the multiplicative Bell parameter is more tightly bounded as follows, $\mathcal{B}_{2} \leqslant 2 \sqrt{1-\eta_{A}^{2}}$ (for derivation please see the Appendix). For both bounds, the local correlation given by $\eta_{A}$ determines the extent of the nonlocal correlations on the right-hand side. It is quite remarkable that the upper bound depends, after all, on a local quantity. These inequalities are derived based on Alice's local correlations, but can be similarly derived for Bob's, and hence an even tighter bound is given by their minimum. To experimentally infer these richer bounds on correlations, we selected two vectors $a_{1}$ and $a_{2}$ on Alice's side that correspond to a particular value of $\eta_{A}$. Then we randomly selected two vectors on Bob's side, and calculated the CHSH and Bell parameters. In Figs. 3(a) and
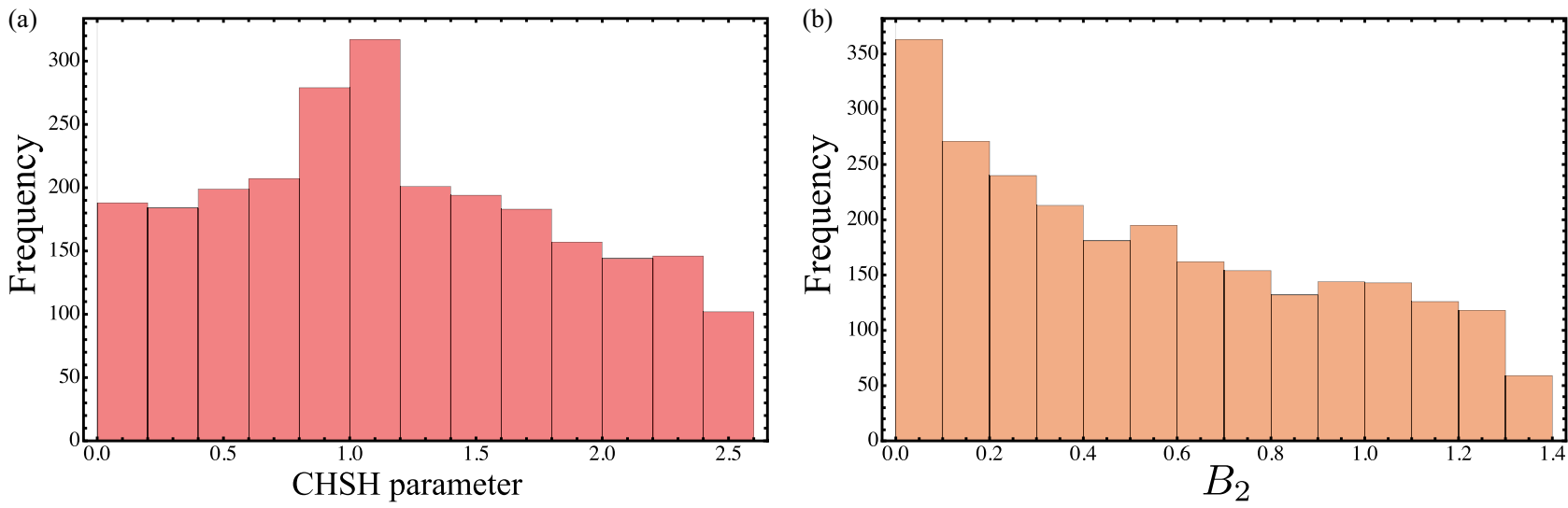

FIG. 3. Distribution of both (a) CHSH parameters (upper limit $=2.618$ ) as well as (b) Bell parameters $\mathcal{B}_{2}$ (upper limit $=1.428$ ) for $\eta_{A}=0.7$. 

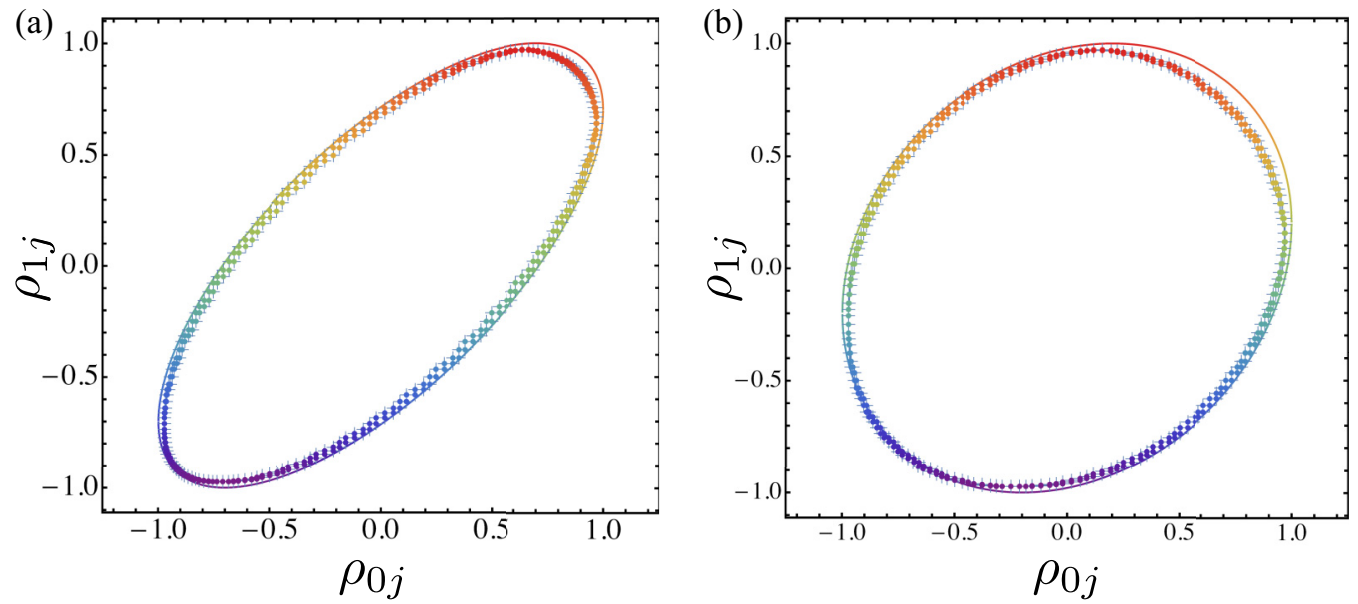

FIG. 4. Distribution of the correlation vectors for (a) $\eta_{A}=0.7$ and (b) $\eta_{A}=0.2$. The solid line indicates the region within which the correlation vectors should fall, and the points are experimentally measured vectors.

3(b), we show the results for both parameters when $\eta_{A}=0.7$. The observed values all fall within the bound as predicted by $\mathrm{CHSH} \leqslant \sqrt{2}\left(\sqrt{1+\eta_{A}}+\sqrt{1-\eta_{A}}\right)$ and $\mathcal{B}_{2} \leqslant 1+\sqrt{1-\eta_{A}^{2}}$, which lends supports to relativistic independence. The local correlations, for example, $\eta_{A}$, also put restrictions on the two particle correlations, which can be defined as

$$
\rho_{i j}=\frac{\left\langle A_{i} B_{j}\right\rangle-\left\langle A_{i}\right\rangle\left\langle B_{j}\right\rangle}{\Delta_{A_{i}} \Delta_{B_{j}}} .
$$

Geometrically, for a particular $\eta_{A}$ the correlation vectors $\left(\rho_{0 j}, \rho_{1 j}\right), j=0,1$, lie on the ellipse whose major and minor axes are related to $\eta_{A}$ as

$$
e_{j}=\sqrt{\left(\frac{1 \pm(-1)^{j}\left|\eta_{A}\right|}{\sqrt{2}}\right)}\left[\begin{array}{c}
1 \\
(-1)^{j}
\end{array}\right] .
$$

Similar relation holds for the vectors $\left(\rho_{i 0}, \rho_{i 1}\right), i=0,1$, defined by the local correlation $\eta_{B}$, on Bob's side. Figure 4 shows the ellipses corresponding to particular values of $\eta_{A}=$ 0.7 , and $\eta_{A}=0.2$, along with the experimentally measured correlation vectors. This is a nonorthodox way of analyzing nonlocality, which may lead to better quantitative understanding thereof.

\section{CONCLUSION}

We derived new classical and quantum bounds for the multiplicative Bell inequalities and experimentally tested them. Our new theoretical results tested herewith, stem from the principle of relativistic independence [22] and hence emphasize the interesting interplay between local and nonlocal correlations. The experimental results show that the multiplicative Bell parameters go beyond their classical limits, thus again falsifying local realism. Additionally, they approach the Tsirelson bound, the upper limit derived from the quantum covariance matrix and the relativistic independence assumption. We were also able to experimentally observe the tighter theoretical bounds on the $\mathrm{CHSH}$ and the Bell parameter $\mathcal{B}_{2}$, lending support to the claim that quantum correlations arise from the locality of uncertainty relations [22]. An additional practical merit of multiplicative Bell inequalities, is that they alleviate the detector efficiency requirement [20] for tests of hidden variable theories. It is challenging for photonic Bell experiments, such as the one reported here, to reach high detection efficiencies, but in principle, multiplicative Bell inequalities, may provide a simple circumvention of the detector efficiency loophole. This was shown to be true in the case of the CHSH inequality [20], which can be violated for efficiencies above $\sim 0.83$, and might be even more valuable if applied to the Clauser-Horne [29] and Eberhard [30] inequalities which can be violated for efficiencies above $2 / 3$. If multiplicative Bell inequalities can be derived for these cases, while maintaining their beneficial treatment of detector efficiency, they may have various applications, e.g., for entanglement-based quantum cryptography.

\section{ACKNOWLEDGMENTS}

The authors would like to thank Alessio D'Erico for fruitful discussions. This work was supported by Canada Research Chairs (CRC), Canada Foundation for Innovation (CFI), Canada First Excellence Research Fund (CFREF), and Grant No. FQXi-RFP-CPW-2006 from the Foundational Questions Institute and Fetzer Franklin Fund, a donor advised fund of Silicon Valley Community Foundation. E.C. acknowledges support from the Israel Innovation Authority under Projects No. 70002 and No. 73795, the Quantum Science and Technology Program of the Israeli Council of Higher Education, and the Pazy Foundation.

\section{APPENDIX}

Here we derive the richer $\mathrm{CHSH}$ and multiplicative Bell inequalities for $n=2$. Let us define the Pearson correlations in a standard Bell-CHSH experiment as

$$
\rho_{i j} \stackrel{\text { def }}{=} \frac{\left\langle A_{i} B_{j}\right\rangle-\left\langle A_{i}\right\rangle\left\langle B_{j}\right\rangle}{\Delta_{A_{i}} \Delta_{B_{j}}},
$$

where the variances are $\Delta_{A_{i}}^{2}=\left\langle A_{i}^{2}\right\rangle-\left\langle A_{i}\right\rangle^{2}$ and $\Delta_{B_{j}}^{2}=$ $\left\langle B_{j}^{2}\right\rangle-\left\langle B_{j}\right\rangle^{2}$. In any theory satisfying the generalized uncertainty relation presented in [22], the principle of relativistic 
causality implies

$$
\begin{aligned}
& {\left[\begin{array}{cc}
1 & \eta_{A} \\
\eta_{A}^{*} & 1
\end{array}\right] \succeq\left[\begin{array}{l}
\rho_{0 j} \\
\rho_{1 j}
\end{array}\right]\left[\begin{array}{ll}
\rho_{0 j} & \rho_{1 j}
\end{array}\right],} \\
& {\left[\begin{array}{cc}
1 & \eta_{B} \\
\eta_{B}^{*} & 1
\end{array}\right] \succeq\left[\begin{array}{l}
\rho_{i 0} \\
\rho_{i 1}
\end{array}\right]\left[\begin{array}{ll}
\rho_{i 0} & \rho_{i 1}
\end{array}\right],}
\end{aligned}
$$

for $i, j \in\{0,1\}$, where $\eta_{A}$ and $\eta_{B}$ are two complex numbers satisfying $\left|\eta_{A}\right| \leqslant 1,\left|\eta_{B}\right| \leqslant 1$, and $\eta^{*}$ denotes the complex conjugate of $\eta$. Quantum mechanics satisfies both relativistic causality and the generalized uncertainty relations [22], for

$$
\eta_{A} \stackrel{\text { def }}{=} \frac{\left\langle A_{0} A_{1}\right\rangle-\left\langle A_{0}\right\rangle\left\langle A_{1}\right\rangle}{\Delta_{A_{0}} \Delta_{A_{1}}}, \quad \eta_{B} \stackrel{\text { def }}{=} \frac{\left\langle B_{0} B_{1}\right\rangle-\left\langle B_{0}\right\rangle\left\langle B_{1}\right\rangle}{\Delta_{B_{0}} \Delta_{B_{1}}} .
$$

It was also shown in [22] that following Tsirelson-like bounds stem from (A2) and (A3),

$$
\begin{aligned}
& |\mathrm{CHSH}| \leqslant \min \\
& \left\{\sqrt{2}\left(\sqrt{1+\operatorname{Re}\left(\eta_{A}\right)}+\sqrt{1-\operatorname{Re}\left(\eta_{A}\right)}\right), \quad 2 \sqrt{2} \sqrt{1-\operatorname{Im}\left(\eta_{A}\right)^{2}}\right\} \\
& \leqslant 2 \sqrt{2},
\end{aligned}
$$

$|\mathrm{CHSH}| \leqslant$ $\min \left\{\sqrt{2}\left(\sqrt{1+\operatorname{Re}\left(\eta_{B}\right)}+\sqrt{1-\operatorname{Re}\left(\eta_{B}\right)}\right), 2 \sqrt{2} \sqrt{1-\operatorname{Im}\left(\eta_{B}\right)^{2}}\right\}$ $\leqslant 2 \sqrt{2}$

where $\mathrm{CHSH} \stackrel{\text { def }}{=} \rho_{00}+\rho_{10}+\rho_{01}-\rho_{11}$ is the Bell-CHSH parameter.

For real $\eta$, Eq. (A4) transforms to

$$
|\mathrm{CHSH}| \leqslant \sqrt{2}\left(\sqrt{1+\eta_{A}}+\sqrt{1-\eta_{A}}\right) \leqslant 2 \sqrt{2},
$$

$$
|\mathrm{CHSH}| \leqslant \sqrt{2}\left(\sqrt{1+\eta_{B}}+\sqrt{1-\eta_{B}}\right) \leqslant 2 \sqrt{2} .
$$

For the multiplicative Bell parameter $\mathcal{B}_{2}$, using the inequality of geometric and arithmetic means and using Eq. (A5a),

$$
\begin{aligned}
& \left|\mathcal{B}_{2}\right|=\left|\left(\rho_{00}+\rho_{10}\right)\left(\rho_{01}-\rho_{11}\right)\right| \leqslant\left(\frac{\rho_{00}+\rho_{10}+\rho_{01}-\rho_{11}}{2}\right)^{2}, \\
& \left|\mathcal{B}_{2}\right| \leqslant \frac{1}{2}\left(\sqrt{1-\eta_{A}}+\sqrt{1+\eta_{A}}\right)^{2}=1+\sqrt{1-\eta_{A}^{2}} .
\end{aligned}
$$

In [21], it was also proven that

$$
\begin{aligned}
& \left|\rho_{00}+\rho_{01}\right| \leqslant \sqrt{2(1+d)}, \\
& \left|\rho_{10}-\rho_{11}\right| \leqslant \sqrt{2(1-d)},
\end{aligned}
$$

where $d=\left\langle\left\{A_{0}, A_{1}\right\}\right\rangle / 2$, and $\left\{A_{0}, A_{1}\right\}$ is the anticommutator of the local operators $A_{0}$ and $A_{1}$. For a maximally entangled state like the singlet state, the expectation values of the local operators are zero, and $d=\eta_{A}$. From Eqs. (A6) and (A7),

$$
\left|\mathcal{B}_{2}\right|=\left|\left(\rho_{00}+\rho_{01}\right)\left(\rho_{10}-\rho_{11}\right)\right| \leqslant 2 \sqrt{1-\eta_{A}^{2}} .
$$

The role of local correlations in determining the nonlocal, Alice-Bob correlations is evident in all these characterizations. Using the above definitions, one may also recognize

$$
\begin{aligned}
& \left|\eta_{A}\right|^{2}=\operatorname{Re}\left(\eta_{A}\right)^{2}+\operatorname{Im}\left(\eta_{A}\right)^{2} \leqslant 1, \\
& \left|\eta_{B}\right|^{2}=\operatorname{Re}\left(\eta_{B}\right)^{2}+\operatorname{Im}\left(\eta_{B}\right)^{2} \leqslant 1
\end{aligned}
$$

as the Schrödinger uncertainty relations of Alice's $A_{0}$ and $A_{1}$, and of Bob's $B_{0}$ and $B_{1}$.
[1] D. Paneru et al., Entanglement: Quantum or classical? Rep. Prog. Phys. 83, 064001 (2020).

[2] A. Einstein, B. Podolsky, and N. Rosen, Can quantummechanical description of physical reality be considered complete? Phys. Rev. 47, 777 (1935).

[3] M. Genovese, Research on hidden variable theories: A review of recent progresses, Phys. Rep. 413, 319 (2005).

[4] J. S. Bell, On Einstein-Podolsky-Rosen paradox, Physics 1, 195 (1964).

[5] N. Brunner, D. Cavalcanti, S. Pironio, V. Scarani, and S. Wehner, Bell non-locality, Rev. Mod. Phys. 86, 419 (2014).

[6] S. J. Freedman and J. F. Clauser, Experimental Test of Local Hidden-Variable Theories, Phys. Rev. Lett. 28, 938 (1972).

[7] A. Aspect, P. Grangier, and G. Roger, Experimental Tests of Realistic Local Theories via Bell's Theorem, Phys. Rev. Lett. 47, 460 (1981).

[8] A. Aspect, P. Grangier, and G. Roger, Experimental Realization of Einstein-Podolsky-Rosen-Bohm Gedankenexperiment: A New Violation of Bell's Inequalities, Phys. Rev. Lett. 49, 91 (1982).

[9] A. Aspect, J. Dalibard, and G. Roger, Experimental Test of Bell's Inequalities using Time-Varying Analyzers, Phys. Rev. Lett. 49, 1804 (1982).
[10] T. Scheidl et al., Violation of local realism with freedom of choice, Proc. Natl. Acad. Sci. USA 107, 19708 (2010).

[11] B. Hensen et al., Loophole-free Bell inequality violation using electron spins separated by 1.3 kilometres, Nature (London) 526, 682 (2015).

[12] M. Giustina et al., Bell violation using entangled photons without the fair-sampling assumption, Nature (London) 497, 227 (2013).

[13] D. Rauch, J. Handsteiner, A. Hochrainer, J. Gallicchio, A. S. Friedman, C. Leung, B. Liu, L. Bulla, S. Ecker, F. Steinlechner et al., Cosmic Bell Test using Random Measurement Settings from High-Redshift Quasars, Phys. Rev. Lett. 121, 080403 (2018).

[14] M. Ansmann et al., Violation of Bell's inequality in Josephson phase qubits, Nature (London) 461, 504 (2009).

[15] M. A. Rowe et al., Experimental violation of a Bell's inequality with efficient detection, Nature (London) 409, 791 (2001).

[16] C. Abellán et al., Challenging local realism with human choices, Nature (London) 557, 212 (2018).

[17] B. S. Cirel'son, Quantum generalizations of Bell's inequality, Lett. Math. Phys. 4, 93 (1980). 
[18] F. Karoly Pál and T. Vértesi, Quantum bounds on Bell inequalities, Phys. Rev. A 79, 022120 (2009).

[19] D. Frustaglia, J. P. Baltanás, C. M. Velázquez-Ahumada, A. Fernández-Prieto, A. Lujambio, V. Losada, and A. Cabello, Classical Physics and the Bounds of Quantum Correlations, Phys. Rev. Lett. 116, 250404 (2016).

[20] A. Te'eni, B. Y. Peled, E. Cohen, and A. Carmi, Multiplicative Bell inequalities, Phys. Rev. A 99, 040102(R) (2019).

[21] A. Carmi and E. Cohen, On the significance of the quantum mechanical covariance matrix, Entropy 20, 500 (2018).

[22] A. Carmi and E. Cohen, Relativistic independence bounds nonlocality, Sci. Adv. 5, eaav8370 (2019).

[23] H. F. Hofmann, Local measurement uncertainties impose a limit on nonlocal quantum correlations, Phys. Rev. A 100, 012123 (2019).

[24] A. Carmi, Y. Herasymenko, E. Cohen, and K. Snizhko, Bounds on nonlocal correlations in the presence of signaling and their application to topological zero modes, New J. Phys. 21, 073032 (2019).

[25] B. Y. Peled, A. Te'eni, D. Georgiev, E. Cohen, and A. Carmi, Double slit with an Einstein-Podolsky-Rosen pair, Appl. Sci. 10, 792 (2020).

[26] E. Cohen and A. Carmi, In praise of quantum uncertainty, Entropy 22, 302 (2020).

[27] J. F. Clauser, M. A. Horne, A. Shimony, and R. A. Holt, Proposed Experiment to Test Local Hidden-Variable Theories, Phys. Rev. Lett. 23, 880 (1969).

[28] R. Rangarajan, M. Goggin, and P. Kwiat, Optimizing type-I polarization-entangled photons, Opt. Express 17, 18920 (2009).

[29] J. F. Clauser and M. A. Horne, Experimental consequences of objective local theories, Phys. Rev. D 10, 526 (1974).

[30] P. H. Eberhard, Background level and counter efficiencies required for a loophole-free Einstein-Podolsky-Rosen experiment, Phys. Rev. A 47, R747 (1993). 